\title{
Scientific goals of Nano-JASMINE
}

\section{Yoshiyuki Yamada, ${ }^{1}$ Sho Fujita,${ }^{1}$ Naoteru Gouda, ${ }^{2}$ Yukiyasu Kobayashi, ${ }^{2}$ Takuji Hara, ${ }^{2,3}$ Ryoichi Nishi, ${ }^{4}$ Satoshi Yoshioka, ${ }^{5}$ Shunsuke Hozumi, ${ }^{6}$ and the JASMINE Working Group}

\author{
${ }^{1}$ Department of Physics, Faculty of Science, Kyoto University, Sakyo-ku, Kyoto 606-8502, \\ Japan \\ email: yamada@scphys.kyoto-u.ac.jp \\ ${ }^{2}$ JASMINE Project Office, National Astronomical Observatory of Japan, 2-21-1 Osawa, \\ Mitaka, Tokyo 181-8588, Japan \\ ${ }^{3}$ Department of Astronomy, School of Science, The University of Tokyo, 7-3-1 Hongo, \\ Bunkyo-ku, Tokyo 113-0033, Japan \\ ${ }^{4}$ Department of Physics, Niigata University, 8050 Ikarashi-2, Niigata 950-2181, Japan \\ ${ }^{5}$ Department of Marine Electronics and Mechanical Engineering, Tokyo University of Marine \\ Science and Technology, 2-1-6 Ecchu-Jima, Koto-ku, Tokyo 135-8533, Japan \\ ${ }^{6}$ Faculty of Education, Shiga University, 2-5-1 Hiratsu, Otsu, Shiga 520-0862, Japan
}

\begin{abstract}
Nano-JASMINE is an ultrasmall Japanese satellite (with a weight of $35 \mathrm{~kg}$ ), designed to carry out an astrometric mission. The target accuracy is 3 milliarcseconds (mas) for stars brighter than magnitude 7.5 at $z w$-band wavelengths of $0.6-1.0 \mu \mathrm{m}$. The observational strategy is the same as that of Gaia and Hipparcos. The time span of 20 years since the Hipparcos mission will enable us to update the proper motion data obtained at that time. With the help of these updated measurements, we expect that some stars will be resolved into multiple stars. In addition, taking advantage of the small primary mirror (with a diameter of $5 \mathrm{~cm}$ ), we can measure bright stars which cannot be observed with Gaia because of saturation limits. The core data reduction for the Nano-JASMINE mission will use Gaia's Astrometric Global Iterative Solution (AGIS). A collaboration between the Gaia AGIS and Nano-JASMINE teams was initiated in 2007.
\end{abstract}

Keywords. astrometry

\section{Introduction}

Nano-JASMINE is a challenging project with as goal astrometric observations of the entire sky using an ultrasmall satellite. The satellite bus system was developed by members of the Department of Aerospace and Aeronautics at the University of Tokyo, while the mission instruments have been developed by members of the National Astronomical Observatory of Japan and Kyoto University. The flight model was constructed in October 2010 (see Fig. 1), and system tests are almost completed. We use an old radio telescope, designed for astronomy, as a ground station to receive scientific data, achieving a downlink speed of $100 \mathrm{kbps}$.

This satellite mission was planned as the second global space astrometric mission. However, it will now become the third mission, following Gaia, since the launch site's construction has been delayed. It will achieve the same order of astrometric accuracy (2-3 mas) as Hipparcos (1 mas). As 20 years have passed since the Hipparcos mission, we will be able to measure proper motions with an order-of-magnitude higher accuracy by combining the data from that time with measurements which will be obtained as part of the current mission. The specifications of the satellite are listed in Table 1. 


\section{Scientific Goals}

Nano-JASMINE will achieve the same order of accuracy as Hipparcos. The accuracy map was derived using the code written by Gaia members Daniel Michalik and Berry Hole. Fig. 2 is a relatively optimistic estimation of the errors. All issues which were taken into account for the Hipparcos data have been considered. Even if Nano-JASMINE's performance would result in lower-accuracy measurements than those of Hipparcos, the number of stars we expect to observe with Nano-JASMINE is approximately $5 \times 10^{5}$, which is approximately $4 \times$ larger than the number of stars observed by Hipparcos $(1.2 \times$ $10^{5}$ ). Consequently, we can expect more refined results than obtained with Hipparcos in relation to some scientific themes, such as constraints on the parameterized postNewtonian (PPN) formalism's $\gamma$ parameter.

By taking advantage of the 20-year baseline, proper motion data will be updated by

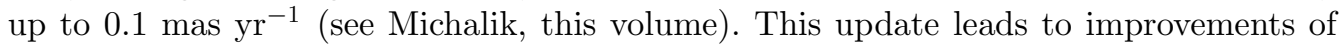
binary parameters, so that we expect that several stars will be identified as binary stars. Such an identification will not be feasible if we depend on either the Hipparcos data alone or the Nano-JASMINE data for 2.5 years of operations. A combination of both data sets is indispensable. Statistical information on kinematic motions of stars is a good tracer of the invisible 'dark matter' through gravity. Nano-JASMINE will achieve unprecedented accuracy for proper motions of stars in the solar neighborhood, enabling us to investigate the nature of dark matter.

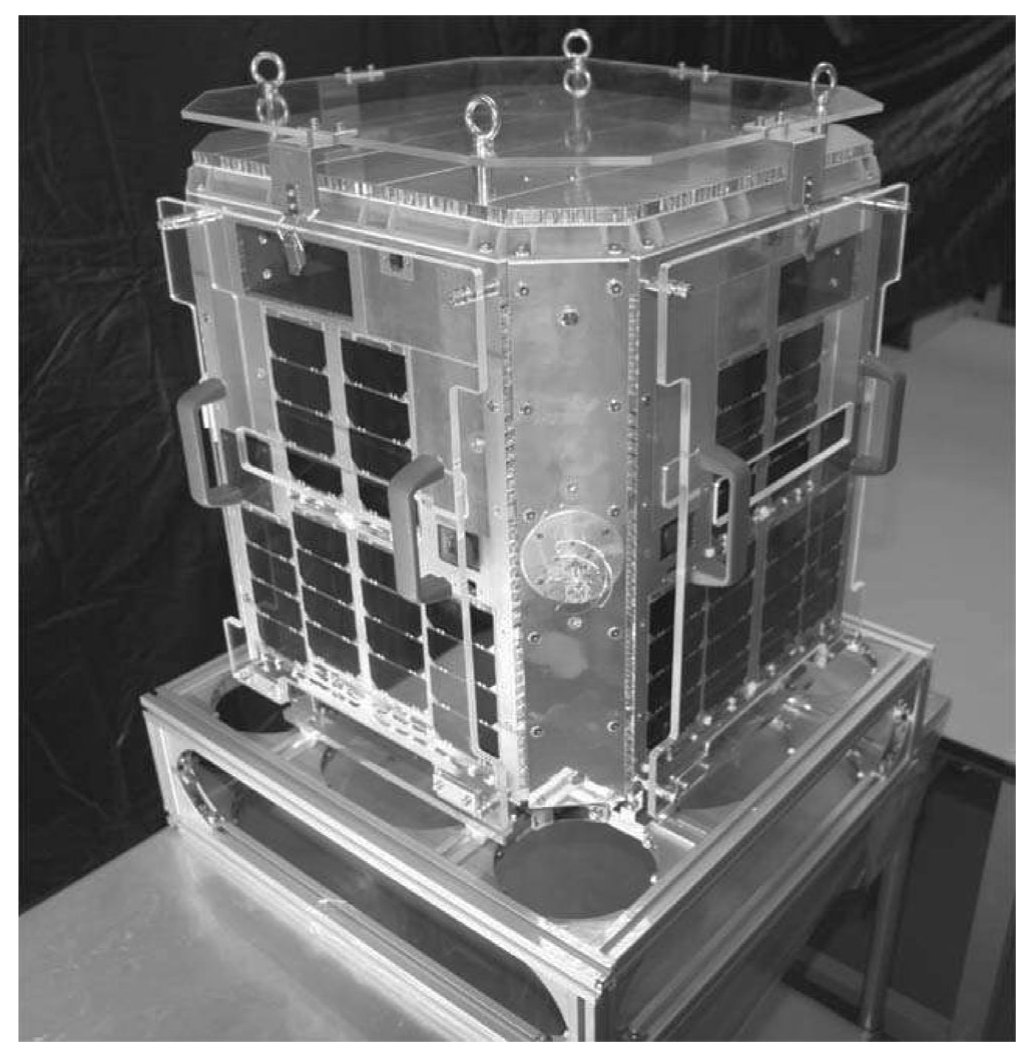

Figure 1. Nano-JASMINE flight model. The satellite was fabricated in October 2010 and its system tests have meanwhile been completed. Now that we have performed the regular 'health checks,' the satellite is ready for launch. 
Table 1. Specifications of the Nano-JASMINE satellite and its catalogues.

\begin{tabular}{|c|c|}
\hline | Size & $50 \mathrm{~cm}$ cubic form \\
\hline Weight & $35 \mathrm{~kg}$ \\
\hline | Launch date & | November 2013-March 2014 \\
\hline |Orbit & | Sun-synchronous orbit at an altitude of $800 \mathrm{~km}$ \\
\hline Obs. wavelength & $\mid z w$ band $(0.6-1.0 \mu \mathrm{m})$ \\
\hline $\begin{array}{l}\text { Aperture size of the } \\
\text { primary mirror }\end{array}$ & $5.25 \mathrm{~cm}$ \\
\hline Focal length & $1.67 \mathrm{~m}$ \\
\hline Detector & Full depletion CCD with $1 \mathrm{k} \times 1 \mathrm{k}$ pixels \\
\hline Number of stars & \begin{tabular}{|l|}
520,000 stars brighter than $\sim 9 \mathrm{mag}$ \\
200,000 stars brighter than $7.5 \mathrm{mag}$
\end{tabular} \\
\hline Position accuracy & |2-3 mas @ < $<.5 \mathrm{mag}, 3-4 \mathrm{mas} @ 9 \mathrm{mag}$ \\
\hline Parallax accuracy & |3-4 mas @ $<7.5 \mathrm{mag}, 4-5 \mathrm{mas} @ 9 \mathrm{mag}$ \\
\hline \multirow[t]{2}{*}{$\begin{array}{l}\text { Proper motion accu- } \\
\text { racy }\end{array}$} & $2-4$ mas yr $^{-1} @<5 \mathrm{mag}, 3-6 \operatorname{mas~yr}^{-1} @ 9 \mathrm{mag}$ \\
\hline & $\begin{array}{l}\sim 0.1 \mathrm{mas} \mathrm{yr}^{-1} @<7.5 \mathrm{mag}\left(\sim 0.2 \mathrm{mas} \mathrm{yr}^{-1} @ 9 \mathrm{mag}\right) \text { combined } \\
\text { with } N-J \text { and Hipparcos catalogs (see Michalik, this volume) }\end{array}$ \\
\hline \multirow[t]{2}{*}{ Catalog } & 2015 proper motion (half-mission data + Hipparcos) \\
\hline & 2016-2017 all astrometric parameters (3 years of data) \\
\hline Comments & | We target stars fainter than $\sim 2$ mag. Other targets: Binaries \\
\hline
\end{tabular}

\section{Data Analysis}

The core data reduction for the Nano-JASMINE mission will be done with Gaia's Astrometric Global Iterative Solution (AGIS). A collaboration between the Gaia agis and Nano-JASMINE teams for the Nano-JASMINE data reduction started in 2007.
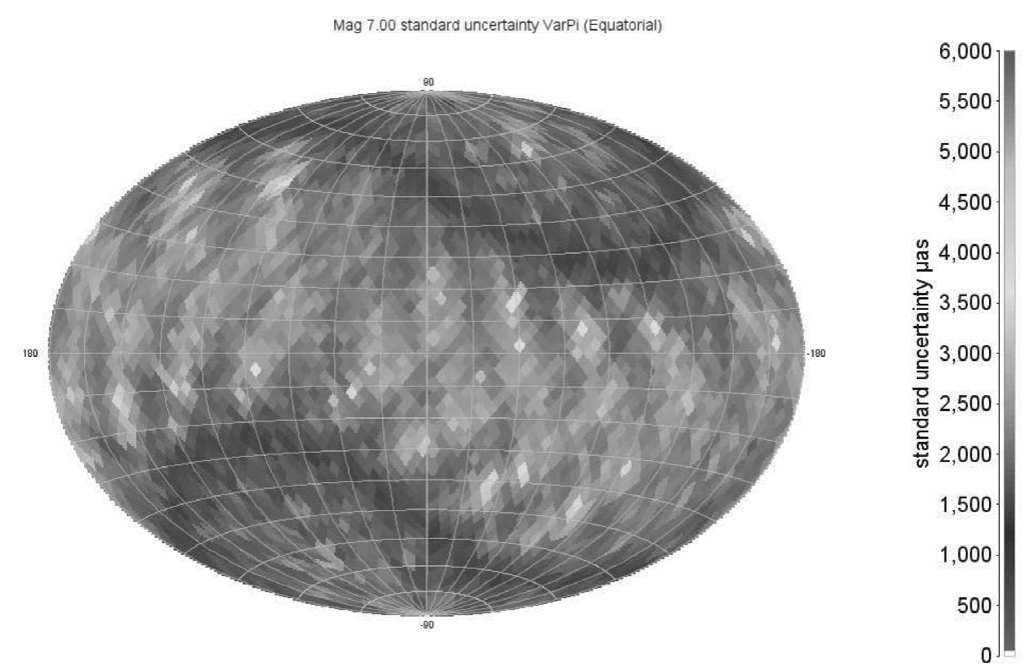

Figure 2. Parallax accuracy map of Nano-JASMINE based on 2 years of observations. 
A data analysis team in Japan performs the IDT (initial data treatment). This includes centroiding and identification of stars. Some members at the Tokyo University of Marine Science and Technology and at Shiga University also contribute to the development of the IDT software. The attitude model is based on a spline model as adopted for Gaia modeling. The orbital modeling is also based on spline interpolation of Global Positioning System data. Radiation damage of the CCD, pixel response, attitude smearing, and charge smearing due to TDI (time-delayed integration) operations have been incorporated in the centroiding tasks (Yamada et al. 2012).

Nano-JASMINE does not have detectors for photometry. This means that we need a reference catalog to obtain the colors of our target stars for astrometric correction. A reference database based on the UCAC3 has been created. We will perform all necessary simulations so that we can estimate the accuracy using this catalog, HILS (Hardware In The Loop Simulator) outputs, and a realistic noise model of the detectors.

Since Nano-JASMINE is supposed to be launched into a Sun-synchronous orbit, we predict that it will suffer from more significant attitude noise than Gaia, which will be operated near the second Lagrange point (L2). We are therefore working on attitude modeling using auto-regressive (AR) models, in collaboration with an IBM Tokyo Research team.

\section{References}

Yamada, Y., Hara, T., Yoshioka, S., et al. 2012, ASP Conf. Ser., 461, 585 